\title{
OBTENCIÓN DE PARÁMETROS DEL MODELO HARDENING SOIL MEDIANTE ENSAYO CPTU EN SUELOS BLANDOS DE BOGOTÁ
}

\author{
Rubby Vanesa Ballesteros Granados ${ }^{1}$
}

${ }^{1}$ Ingeniera civil. Universidad Pedagógica y Tecnológica de Colombia UPTC. Grupo de Investigación GIISAG. Tunja-Colombia.rubby.ballesteros@uptc.edu.co

\section{RESUMEN}

Proyectos de ingeniería como excavaciones, cimentaciones y/o estabilización de taludes, cada día requieren estudios más exigentes que implementen análisis en elementos finitos para obtener resultados más precisos y confiables, esto sólo es posible garantizando la calidad de la información utilizada y definiendo adecuadamente el modelo constitutivo que mejor represente el comportamiento del suelo. Ya sea el modelo Mohr Coulomb, Cam Clay, Hardening Soil u otros disponibles, la obtención de parámetros de entrada con técnicas convencionales de exploración requieren de ejecutar múltiples ensayos que en algunas ocasiones resultan costosos. Por esta razón, los ensayos con técnicas avanzadas de exploración como la prueba de penetración de cono con medición de presión de poros CPTu, representa una buena alternativa para caracterizar el suelo de forma rápida y detallada. Este artículo presenta la obtención de parámetros del modelo Hardening Soil a partir del ensayo CPTu y su validación con resultados de ensayos de laboratorio.

Palabras clave: Elementos finitos, Modelo constitutivo, CPTu, Modelo Hardening soil.

Recibido: 16 de Agosto de 2017.

Received: August 16th, 2017.
Aceptado: 2 de Mayo de 2018

Accepted: May 2nd, 2018

\section{OBTAINING PARAMETERS OF THE HARDENING SOIL MODEL BY TESTING CPTU ON SOFT SOILS OF BOGOTÁ}

\begin{abstract}
Engineering projects such as excavations, foundations and / or slope stabilization require more demanding studies that implement finite element analysis to obtain more accurate and reliable results, this is only possible by guaranteeing the quality of the information used and adequately defining the constitutive model that best represents the behavior of the soil. Whether the Mohr Coulomb model, Cam Clay, Hardening Soil or others available, the obtaining of input parameters with conventional exploration techniques performing multiple tests that are sometimes costly. For this reason, tests with advanced exploration techniques such as cone penetration test with pore pressure measurement CPTu, represent a good alternative to characterize the soil quickly and in detail. This article presents the obtaining of parameters for the model Hardening soil based on the CPTu test and validation with results of laboratory tests.
\end{abstract}

Keywords: Finite elements, Constitutive model, CPTu, Hardening soil model.

Cómo citar este artículo: R. V. Ballesteros Granados, "Obtención de Parámetros del Modelo Hardening Soil mediante Ensayo CPTu en Suelos Blandos De Bogotá", Revista Politécnica, Año 14, Vol 14, No 26, pp 8997, 2018. https://doi.org/10.33571/rpolitec.v14n26a8 


\section{INTRODUCCIÓN}

Para la ejecución de cualquier proyecto de ingeniería, primero se debe conocer las características físicas y mecánicas del perfil del suelo sobre el cual se realizará el proyecto, las cuales dependerán del tipo de material, ambiente externo, exposición etc. Es por ello que el método de exploración toma un papel muy importante en la caracterización del suelo, ya que debe proporcionar información precisa de la zona de estudio, y además debe satisfacer las inquietudes del propietario relacionadas con los costos, por esta última razón en múltiples ocasiones para proyectos individuales de menor impacto, se realizan caracterizaciones geotécnicas muy económicas las cuales no garantizan la calidad de los datos obtenidos [1].

Para proyectos con mayor impacto, se entiende la necesidad de realizar estudios detallados y análisis con modelos constitutivos con el fin de evitar problemas futuros por falta de información o errores en el diseño de las obras. Para suelos blandos altamente comprensibles como los de la sabana de Bogotá [2], cuando se utilizan técnicas convencionales de exploración [3], la caracterización de un modelo constitutivo como el Hardening Soil [4], se vuelve costosa al requerir entre otros parámetros, rigidez secante de ensayos tríaxiales $E_{50}$, la rigidez en descarga y recarga $E_{u r}, y$ Rigidez tangente para carga primaria edométrica $E_{o e d}$, de cada uno de los estratos de suelo; la veracidad de esta información dependerá de la calidad de las muestras recuperadas, y de la calidad de ensayos realizados, es por ello que para aumentar la precisión en la información del perfil geotécnico se implementan técnicas avanzadas de exploración y caracterización del subsuelo con ensayos como el de penetración estática con medición de presión de poros CPTu entre otros, que brindan información estratigráfica más detallada y continua, y adicionalmente se desarrollan en un menor tiempo [3].

En el presente artículo se determinan los parámetros básicos del modelo Hardening Soil para el sector de la carrera 9 con calle 127 en la ciudad de Bogotá D.C, mediante los resultados de un ensayo CPTu; los datos son validados con los resultados de un sondeo mecánico realizado hasta alcanzar una profundidad de $50 \mathrm{~m}$. La información es tomada con base en los estudios y diseños de la primera línea del metro tramo IV [5].

\section{MATERIALES Y METODOS}

\subsection{Localización}

La caracterización se realiza en los suelos blandos del norte de la ciudad de Bogotá, en la carrera $9^{a}$ con calle 127 Fig.1, sobre el trazado de la línea del metro tramo IV, para suelos altamente compresibles [2].

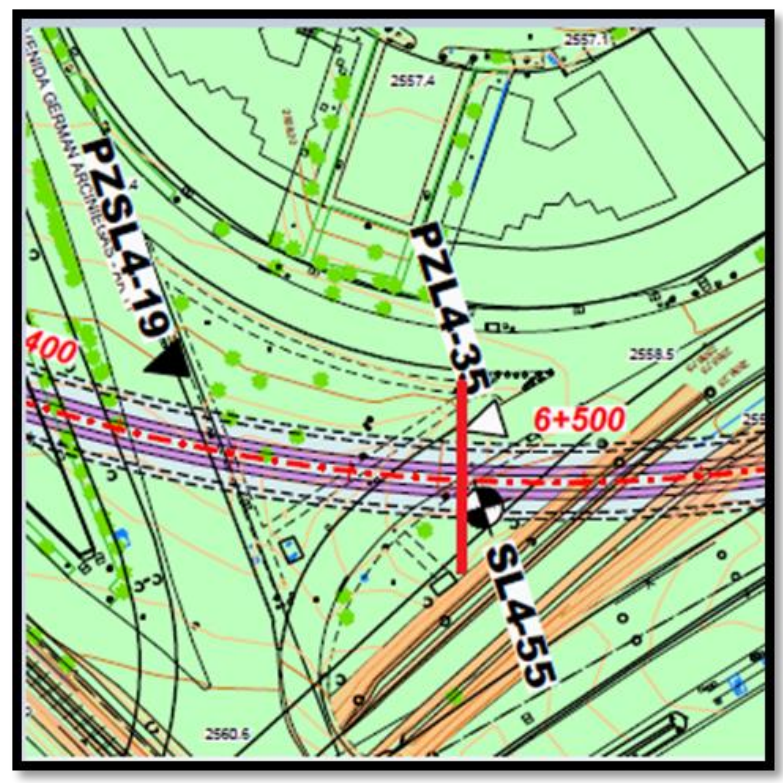

Fig.1. Localización zona de estudio carrera 9ª con calle 127.

\subsection{Recopilación de información disponible}

Para el sector Norte de la ciudad de Bogotá en la carrera 9a con calle 127 , se toma la información del sondeo mecánico SL 4-55 el cual alcanzó una profundidad de $50 \mathrm{~m}$, y del registro CPTu PZL4-35, el cual alcanzó una profundidad de $36 \mathrm{~m}$ [5].

En los $50 \mathrm{~m}$ de perforación se encuentran suelos clasificados como arcillosos de alta plasticidad, limos con presencia de algunas arenas finas, materia orgánica y arcillas de consistencia blanda a muy blanda [5]. En este sondeo se obtuvo muestras alteradas y muestras inalteradas para las cuales se realizaron ensayos de clasificación, resistencia y deformabilidad con pruebas de laboratorio tales como Densidad, Masa unitaria, Gravedad específica, Límites de Atterberg, Corte directo, 
Compresión inconfinada, Tríaxial y Bender Element. La Tabla 1, presenta la cantidad y la profundidad de ensayos realizados. Por su parte, la Fig.2, presenta la comparación y variación de algunos de estos parámetros respecto a la profundidad.

Tabla 1. Cantidad de ensayos realizados [5]

\begin{tabular}{lr}
\hline \multicolumn{1}{c}{ Parámetro } & Cantidad \\
\hline vsat $\left(\mathrm{kN} / \mathrm{m}^{3}\right)$ & 9 \\
$\mathrm{rd}\left(\mathrm{kN} / \mathrm{m}^{3}\right)$ & 9 \\
wn $(\%)$ & 11 \\
Gs & 6 \\
Límite Líquido & 10 \\
Límite Plástico & 10 \\
Índice de plasticidad & 10 \\
Compresibilidad & \\
Consolidación Unidimensional & 1 \\
Resistencia & \\
Compresión inconfinada & 5 \\
Compresión Tríaxial CU & 1 \\
Bender element & 2
\end{tabular}

Fuente: El autor

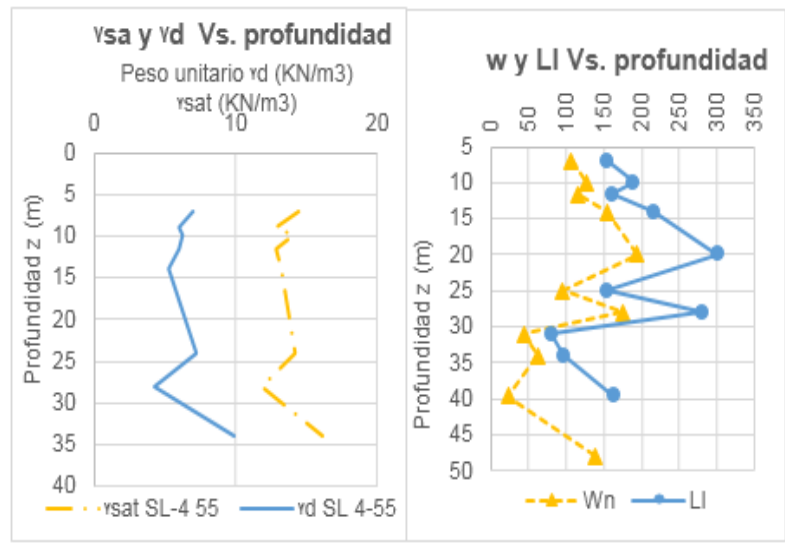

Fig.2. a) rsat y rd vs $Z$, b) Wn y LL vs $Z$

La Fig.2a, muestra el comportamiento del peso unitario, los cuales se obtuvieron mediante ensayos convencionales, se observa que el peso unitario saturado inicia en $14 \mathrm{kN} / \mathrm{m}^{3}$, para una profundidad de 7 metros, y en 34 metros alcanza un valor máximo de $16 \mathrm{kN} / \mathrm{m}^{3}$ en un suelo identificado como arcilla de alta plasticidad, el peso unitario posteriormente disminuye en un lente de turba a una profundidad de $28 \mathrm{~m}$. El peso unitario seco alcanza un valor máximo de $9.9 \mathrm{kN} / \mathrm{m}^{3}$, a una profundidad de $34 \mathrm{~m}$, y el valor menor corresponde a $4.3 \mathrm{kN} / \mathrm{m}^{3}$, a $28 \mathrm{~m}$ de profundidad

En la Fig.2b, se observa que la humedad natural no supera el límite líquido, además presenta un comportamiento variable para el caso de humedad natural en donde se alcanzan valores de 195\% para un suelo identificado como limo orgánico a una profundidad de $20 \mathrm{~m}$, y $23 \%$ para suelo de arena fina a $40 \mathrm{~m}$; El valor máximo y mínimo de límite líquido corresponde a $301 \%$ y $80 \%$ respectivamente.

Con los datos del CPTu PZL4-35, se determinan los estratos del suelo y sus respectivos espesores, teniendo en cuenta la variación del Índice de comportamiento lc en función de la profundidad como se muestra en la Fig.3, [6].

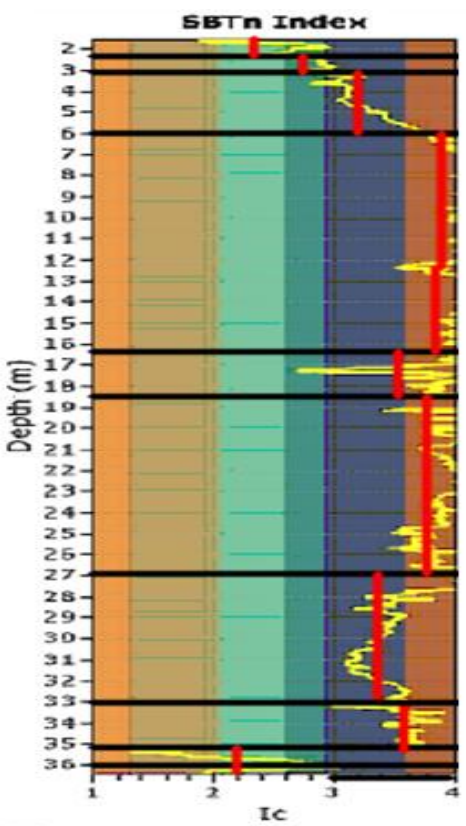

Fig.3. Definición estratigrafía y determinación de lc promedio.

Una vez determinados los espesores, estos se relacionan con la descripción in situ obtenida del sondeo mecánico. La Tabla 2, presenta la profundidad y la descripción in situ del suelo. 
Tabla 2. Descripción in situ del suelo

\begin{tabular}{|c|c|}
\hline $\begin{array}{c}\mathrm{Z} \\
(\mathrm{m})\end{array}$ & Descripción \\
\hline 2 & Relleno antrópico con arena limosa. \\
\hline 3 & Arcilla con indicios de limo y materia orgánica. \\
\hline 6 & $\begin{array}{l}\text { Arcilla limosa blanda, con presencia de } \\
\text { materia orgánica }\end{array}$ \\
\hline 17 & $\begin{array}{l}\text { Arcilla de alta plasticidad con presencia de } \\
\text { materia orgánica muy blanda. }\end{array}$ \\
\hline 18 & $\begin{array}{l}\text { Arcilla limosa con presencia de materia } \\
\text { orgánica muy blanda. }\end{array}$ \\
\hline 27 & $\begin{array}{l}\text { Arcilla y limo arcilloso de alta plasticidad con } \\
\text { indicios de arena. }\end{array}$ \\
\hline 34 & $\begin{array}{l}\text { Arcilla y limo orgánico, con presencia de } \\
\text { materia orgánica. }\end{array}$ \\
\hline 35 & Limo con presencia de arena y arena limosa. \\
\hline 37 & Arena limosa \\
\hline
\end{tabular}

Fuente: El Autor

\subsection{Parámetros del modelo Hardening Soil.}

Los parámetros necesarios para caracterizar el modelo Hardening Soil se resumen en la Tabla 3 [7].

Tabla 3. Parámetros modelo Hardening Soil

\begin{tabular}{|c|c|}
\hline Descripción & Nomenclatura \\
\hline Peso unitario & $\gamma \mathrm{kN} / \mathrm{m}^{2}$ \\
\hline Cohesión & $c^{\prime} \mathrm{kPa}$ \\
\hline Ángulo de fricción & $\Phi\left({ }^{\circ}\right)$ \\
\hline Ángulo de dilatancia & $\Psi\left({ }^{\circ}\right)$ \\
\hline $\begin{array}{l}\text { Rigidez tangente para carga } \\
\text { primara }\end{array}$ & $\mathrm{E}_{\mathrm{oed}}{ }^{\text {ref }} \mathrm{MPa}$ \\
\hline $\begin{array}{l}\text { Rigidez secante en ensayos } \\
\text { tríaxiales }\end{array}$ & $\mathrm{E}_{50}{ }^{\text {ref }} \mathrm{MPa}$ \\
\hline Rigidez en descarga y recarga & $E_{u r}{ }^{\text {ref }} \mathrm{MPa}$ \\
\hline $\begin{array}{l}\text { Dependencia de la rigidez al } \\
\text { estado de esfuerzos }\end{array}$ & $m$ \\
\hline
\end{tabular}

Fuente: El Autor

\subsection{Metodología de determinación de parámetros}

La Tabla 4, presenta la fuente a partir de la cual se determinaron los parámetros del modelo Hardening Soil, los que se determinaron por medio del CPTu se validaron con ensayos de laboratorio.

Tabla 4. Fuente de determinación de parámetros modelo Hardening Soil

\begin{tabular}{|c|c|c|}
\hline Parámetro & $\begin{array}{c}\text { Método de } \\
\text { determinación }\end{array}$ & $\begin{array}{l}\text { Método de } \\
\text { validación }\end{array}$ \\
\hline$\gamma \mathrm{kN} / \mathrm{m}^{2}$ & CPTu & Ensayo Laboratorio \\
\hline$c^{\prime} \mathrm{kPa}$ & - & Otros autores \\
\hline$\Phi\left(^{\circ}\right)$ & CPTu & Ensayo Laboratorio \\
\hline$\Psi\left({ }^{\circ}\right)$ & - & Otros autores \\
\hline $\mathrm{E}_{\text {oed }}{ }^{\text {ref }} \mathrm{MPa}$ & CPTu & Ensayo Laboratorio \\
\hline $\mathrm{E}_{50}{ }^{\text {ref }} \mathrm{MPa}$ & Correlación & Otros autores \\
\hline Eur ${ }^{\text {ref }} \mathrm{MPa}$ & Correlación & Otros autores \\
\hline$m$ & $\begin{array}{c}\text { Fuente } \\
\text { Académica }\end{array}$ & Ensayo Laboratorio \\
\hline
\end{tabular}

Fuente: El Autor

En la Ecuación 1, se presenta el cálculo del peso unitario del suelo en función de la resistencia por fuste $\mathrm{Rf}$ y resistencia por punta corregida qt, obtenidos del registro CPTu [8].

Por medio del ensayo CPTu, se determinan los parámetros de ángulo de fricción $\Phi$, y modulo rigidez tangente para carga Edométrica, Eoed teniendo en cuenta la relación entre $\mathrm{M}=$ Eoed [9]. Posteriormente se calculan los parámetros de referencia Eoedref, E50ref y Eurref con las Ecuaciones 3-5 [4].

El parámetro $\mathrm{m}$ o rigidez dependiente del esfuerzo, es obtenido de la Fig.4, con las gráficas en función del Límite Líquido e Índice de Plasticidad [10] y partiendo de [4], en donde $m=1$ para suelos blandos. 


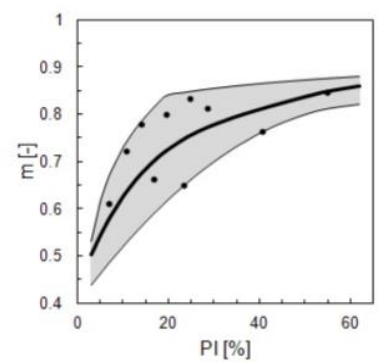

(a)

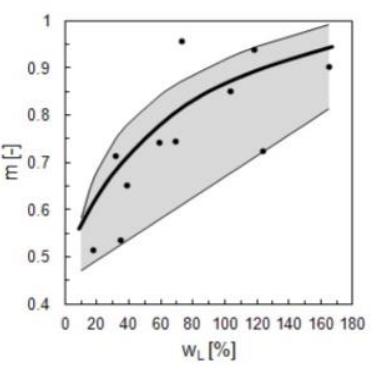

(b)
Fig.4. Relación de ley de potencia m, a) Índice de plasticidad, b) Límite líquido. [10]

El ángulo de dilatancia $\Psi$, es tomado como 0 , ya que los suelos arcillosos tienden a no presentar dilatancia. En la mayoría de los casos $\psi=0$ para valores menores de $\Phi=30^{\circ}[4]$.

Peso unitario del suelo

$\gamma t=Y_{w} *\left(0.27 \log R_{f}+0.36 \log \left(\frac{q_{t}}{P_{a}}\right)+1.236\right)$

Rigidez tangente para carga primaria, con Pref $=$ $100 \mathrm{kPa}$.

$E_{\text {oed }}=E_{\text {oed }}^{r e f}\left(\frac{\sigma}{\text { pref }}\right)^{m}$

Rigidez secante en ensayos tríaxiales.

$E_{\text {oed }}^{r e f} \approx E_{50}^{r e f}$

Rigidez en descarga y recarga de referencia.

$E_{u r}^{R r e f}=3 E_{50}^{r e f}$

\section{Cohesión}

En las arcillas de la Sabana de Bogotá se ha encontrado el siguiente rango en los parámetros de resistencia en término de esfuerzos efectivos y totales. [11].

$c^{\prime}=0-45 \mathrm{kN} / \mathrm{m}^{2}, \Phi=20$ y $46^{\circ}$

$\mathrm{C}=10-50 \mathrm{kNm}^{2} . \Phi=10$ y $20^{\circ}$
Además se recomienda que $\mathrm{c}>0.2 \mathrm{kPa}$. [4], para evitar errores en el momento de la modelación con el modelo Hardening Soil.

\subsection{Determinación de parámetros modelo Hardening Soil estrato No4.}

Como ejemplo aplicativo, se presentan los resultados de la obtención de parámetros del estrato 4 a una profundidad de $11.6 \mathrm{~m}$.

\section{Peso unitario del suelo}

El peso unitario del suelo se calcula por medio del ensayo CPTu, y se compara con el ensayo convencional, de lo cual se observa que se obtiene un resultado ligeramente mayor utilizando el CPTu.
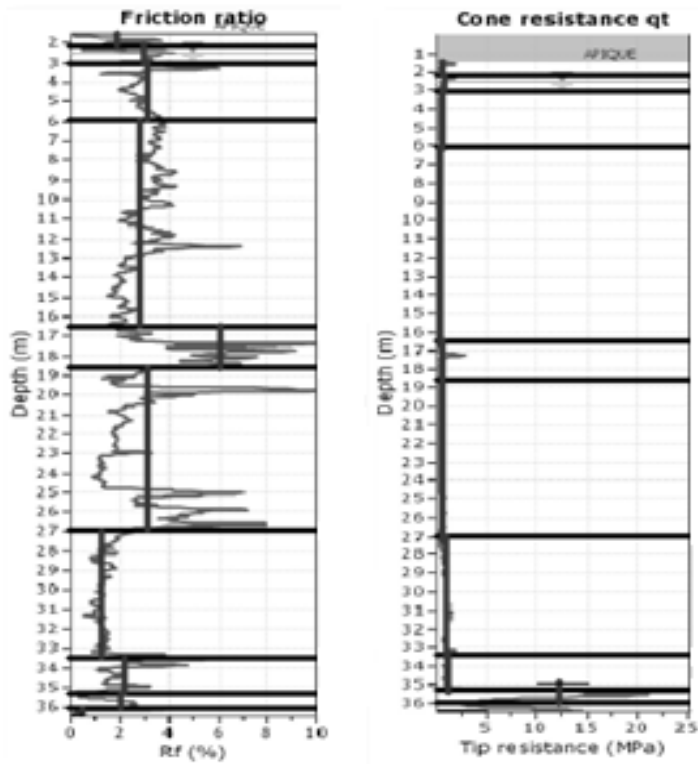

Fig.5. Resistencia por fuste Rf (\%) y resistencia por punta corregida qt.

De la Fig.5, se obtuvo a $11.6 \mathrm{~m}$ que la resistencia por fuste $\mathrm{Rf}=3 \%$ y la resistencia por punta corregida qt $=100 \mathrm{kPa}$, por tanto reemplazando Ecuación 1, el valor del peso unitario del suelo corresponde a $13.64 \mathrm{kN} / \mathrm{m}^{3}$.

Por otra parte el valor de peso unitario de Fig.2, con ensayos convencionales $\mathrm{Y}=13.2 \mathrm{kN} / \mathrm{m}^{3}$. 


\section{Cohesión}

Para determinar el valor de la cohesión se toma en cuenta las recomendaciones en [11], por ello, para suelos blandos del sector Norte de la ciudad de Bogotá, se le asigna un valor conservador de 10 $\mathrm{kPa}$.

\section{Ángulo de fricción $E_{50}{ }^{R e f}, E_{o e d}{ }^{\text {ref }} y E_{u r}{ }^{\text {ref. }}$.}
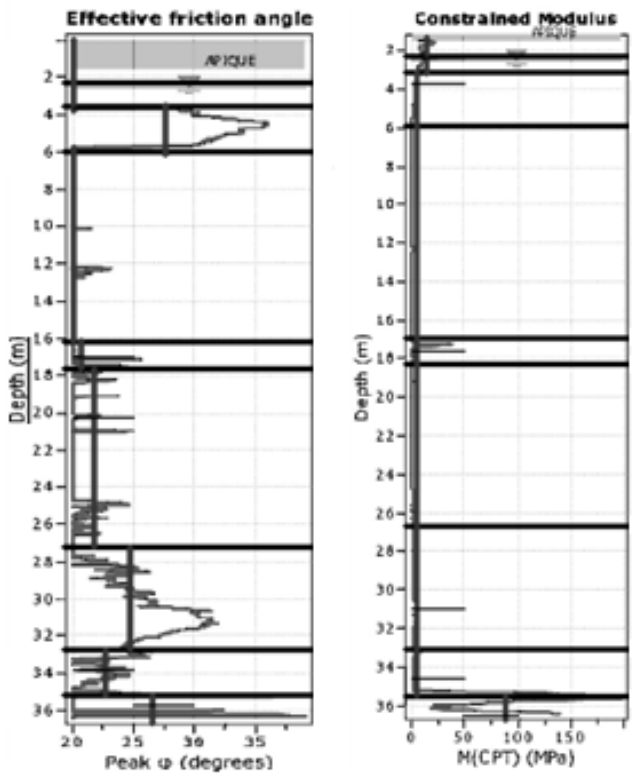

Fig.6. Ángulo de fricción y M ensayo CPTu

De la Fig.6, se obtuvo $\Phi=20^{\circ}, \mathrm{M}=3 \mathrm{MPa}$, el esfuerzo efectivo en el estrato 4 corresponde a $0.0533 \mathrm{MPa}$, por tanto, empleando la Ecuación 2, $E_{50}{ }^{\text {ref }}=5.63 \mathrm{MPa}$, por tanto $E_{o e d}{ }^{\text {ref }}=5.62 \mathrm{Mpa} y$ $\mathrm{E}_{\mathrm{ur}}^{\mathrm{ref}}=16.87 \mathrm{MPa}$.

\section{Dilatancia}

El estrato 4, corresponde a una arcilla de alta plasticidad con presencia de materia orgánica muy blanda. Adicionalmente el ángulo de fricción $\Phi$ es de $20^{\circ}$, por tanto $\Phi<30^{\circ}$, de esta forma se establece para suelos blandos [4] $\Psi=0$.

\section{Parámetro $m$, Dependencia de la rigidez al estado de esfuerzos}

De acuerdo con la Fig.4, para un límite líquido de $175 \%$ correspondiente al estrato $4, \mathrm{~m}=0.9987$.

\subsection{Validación de información de CPTu con sondeo mecánico.}

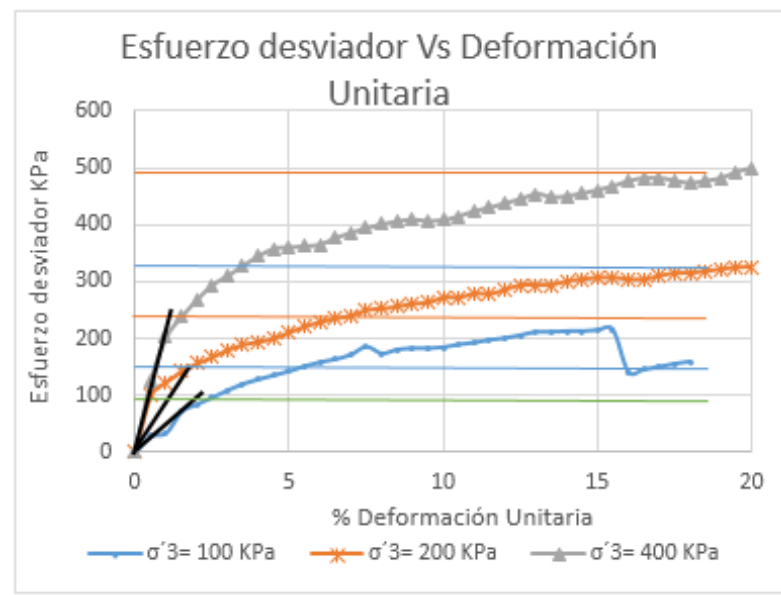

Fig.7. Esfuerzo desviador vs Deformación

La Fig.7, muestra los resultados de un ensayo tríaxial $\mathrm{CU}$, en donde tres muestras de suelo obtenidas a una profundidad de $11.6 \mathrm{~m}$ correspondiente al estrato 4, fueron sometidas a una presión de cámara de $100 \mathrm{kPa}, 200 \mathrm{kPa}$ y de $400 \mathrm{kPa}$

La rigidez secante de ensayos tríaxiales $\left(E_{50}\right)$, se determina con ayuda de la Fig.7, por medio del trazado de una línea desde el origen 0.0 al punto secante al $50 \%$, de esta manera se toma la lectura de deformación y del esfuerzo desviador para cada una de las muestras, tal como se resume en la Tabla 5.

Tabla 5. E50 y Esfuerzo de confinamiento.

\begin{tabular}{llll}
\hline$\sigma 3 \mathrm{kPa}$ & 400 & 200 & 100 \\
$\mathrm{E}_{50} \mathrm{MPa}$ & 14.4 & 7.96 & 3.1 \\
\hline
\end{tabular}

Posteriormente se calcula $E_{50}$ para el esfuerzo de referencia de $100 \mathrm{kPa}$ y el $E_{50}$ de referencia según las Ecuaciones 2 y 3.

$$
E_{50}=\frac{q}{\varepsilon}=\frac{1}{0.032}=31.25 \frac{\mathrm{Kg}}{\mathrm{cm} 2}=3.1 \mathrm{MPa}
$$


$E_{50}^{r e f}=E_{\text {oed }}^{r e f}=\frac{3.1 \mathrm{Mpa}}{\left(\frac{0.0533 \mathrm{Mpa}}{0.1 \mathrm{Mpa}}\right)^{1}}=5.81 \mathrm{MPa}$

Se puede observar que el valor de $E_{50}$ ref para un esfuerzo de referencia de $100 \mathrm{kPa}$ corresponde a $5.81 \mathrm{MPa}$, este valor se asemeja al obtenido por medio del CPTu, el cual es de $5.63 \mathrm{MPa}$, Fig.6, por tal razón, se consideran aceptables los valores obtenidos en las gráficas del CPTu.

Para el cálculo de la dependencia de la rigidez al estado de esfuerzos o parámetro $m$, se grafica $E_{50}$ vs Esfuerzo Desviador [7], con los datos presentes en la Tabla 4, tal como se ilustra en la Fig.8.

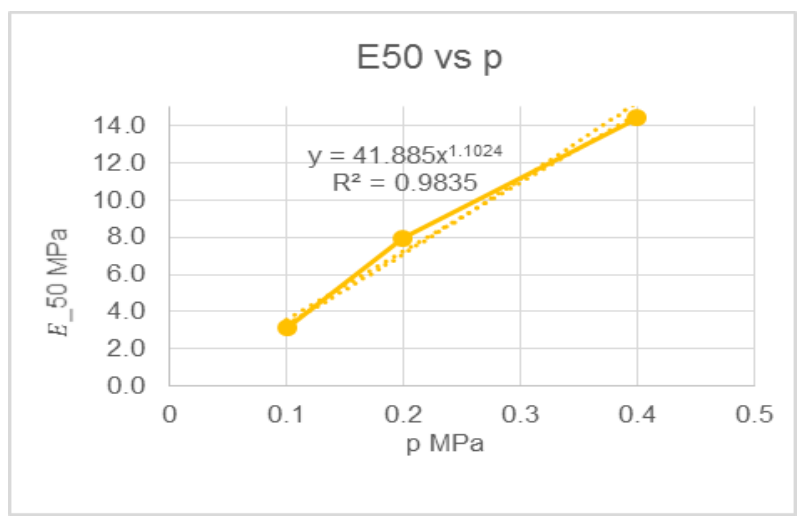

Fig.8. Rigidez dependiente del esfuerzo $\mathrm{m}=1$

La Fig.9, presenta los círculos de Mohr obtenidos para el ensayo tríaxial, en donde se encuentra un valor de ángulo de fricción de $19.28^{\circ}$, el cual se compara el obtenido con el CPTu de la Fig.6, el cual corresponde a $20^{\circ}$.

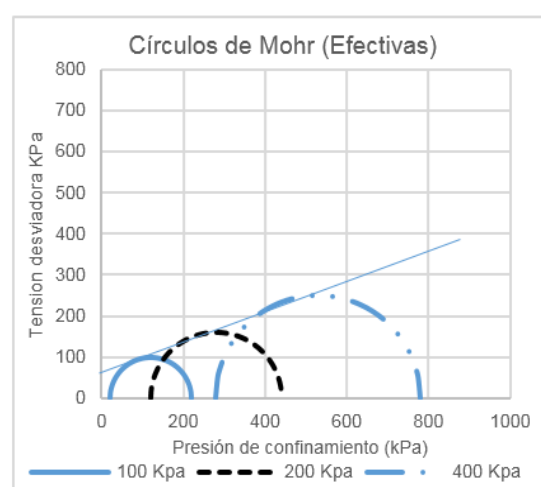

Fig.9. Tensión Desviadora vs \% Deformación, donde el ángulo de fricción $\Phi=19.28^{\circ}$.

\section{RESULTADOS}

Tabla 6. Parámetros modelo Hardening Soil.

\begin{tabular}{llccccc}
\hline $\mathbf{Z}(\mathbf{m})$ & $\begin{array}{l}\mathbf{k N} / \mathbf{m}^{3} \\
\end{array}$ & $\left.\boldsymbol{\Phi} \mathbf{(}^{\circ}\right)$ & $\begin{array}{l}\mathbf{E}_{\text {oed }} \\
\mathbf{M P a}\end{array}$ & $\begin{array}{l}\mathbf{E}_{50} \text { ref } \\
\mathbf{M P a}\end{array}$ & $\begin{array}{c}\mathbf{E}_{\text {ur }}^{\text {ref }} \\
\mathbf{M P a}\end{array}$ & $\boldsymbol{M}$ \\
\hline 0 a 2 & 14 & 20.08 & 28.18 & 28.18 & 84.55 & 0.5 \\
3 & 14 & 20.08 & 29.05 & 29.05 & 87.16 & 0.857 \\
6 & 13.5 & 27.56 & 9.47 & 9.47 & 28.42 & 0.857 \\
17 & 13.6 & 20 & 5.63 & 5.63 & 16.88 & 1 \\
18 & 13.2 & 20.54 & 5.28 & 5.28 & 15.84 & 1 \\
27 & 14.2 & 21.6 & 5.63 & 5.63 & 16.88 & 0.999 \\
34 & 11.8 & 24.7 & 3.48 & 3.48 & 10.45 & 0.845 \\
36 & 16 & 22.58 & 3.21 & 3.21 & 9.63 & 0.86 \\
37 & 16 & 26.58 & 57.54 & 57.54 & 172.63 & 0.5 \\
\hline
\end{tabular}

Con $\Psi=0$ y $c^{\prime}=10 \mathrm{kPa}$.

La Tabla 6 presenta los parámetros básicos de caracterización del modelo constitutivo Hardening Soil, obtenidos con los datos del ensayo CPTu PZL4-35, para un perfil que llega hasta $36 \mathrm{~m}$ de profundidad, estratificado en 9 capas.

Por otra parte, la Tabla 7, presenta los resultados comparativos a una profundidad promedio de $17 \mathrm{~m}$, de los parámetros obtenidos por medio del ensayo CPTu, y de los ensayos de laboratorio; la dependencia de la rigidez al estado de esfuerzos $m$, se determinó por medio del empleo de la Fig. 4, y el parámetro $\Psi$ se determinó con base en las características del suelo por recomendaciones dadas en la literatura.

Tabla 7. Comparativo de resultados modelo Hardening Soil.

\begin{tabular}{cccc}
\hline Parámetro & CPTu & Laboratorio & Correlación \\
\hline $\mathrm{Y} \mathrm{kN} / \mathrm{m}^{3}$ & 13.64 & 13.2 & No aplica \\
$\Phi\left(^{\circ}\right)$ & 20 & 19.28 & No aplica \\
$\mathrm{E}_{\text {oed }} \mathrm{MPa}$ & 3 & 3.1 & No aplica \\
Eoed $^{\text {ref }} \mathrm{MPa}$ & 5.63 & 5.81 & No aplica \\
E $_{50^{\text {ref }}} \mathrm{MPa}$ & 5.63 & 5.81 & No aplica \\
Eur $_{\text {ref }} \mathrm{MPa}$ & 16.89 & 17.43 & No aplica \\
$m$ & & 1 & 1 \\
\hline
\end{tabular}




\section{DISCUSIÓN RESULTADOS)}

$(0$

ANÁLISIS

DE

El valor de peso unitario obtenido por medio de la Ecuación 1, corresponde a $\mathrm{y}=13.64 \mathrm{kN} / \mathrm{m}^{3}$, y el del ensayo convencional es de $\mathrm{Y}=13.2 \mathrm{kN} / \mathrm{m}^{3}$, los cuales difieren en un $3.2 \%$.

Se evidencia que el ángulo de fricción obtenido con el registro del CPTu corresponde a $\Phi=20^{\circ}$, y es un valor muy cercano al obtenido con el ensayo tríaxial el cual corresponde a $\Phi=19.28^{\circ}$.

La rigidez secante de ensayos tríaxiales $\mathrm{E}_{50}$, que se obtiene con la lectura directa de la Fig.6, de los registros del registro CPTu, presenta un valor de rigidez secante $E_{50}=3 \mathrm{MPa}$, por otro lado, con el ensayo tríaxial $\mathrm{E}_{50}=3.1 \mathrm{MPa}$, estos dos valores se asemejan, y corresponden a $E_{50}{ }^{\text {ref }}=5.63 \mathrm{MPa}$ y $\mathrm{E}_{50}{ }^{\text {ref }}=5.81 \mathrm{MPa}$, respectivamente.

El parámetro $m$, o dependencia de la rigidez al estado de esfuerzos, es de uno (1), al realizar la lectura de $m$, con el límite líquido Fig.4, esta información se valida en la Fig.8 con la gráfica de $E_{50}$ vs Esfuerzo Desviador.

\section{CONCLUSIONES}

El ensayo de penetración estática con medición de presión de poros CPTu, permite determinar con alta confiabilidad los parámetros básicos del modelo constitutivo Hardening Soil en suelos Blandos.

Una limitación del ensayo CPTu en la determinación de parámetros del modelo Hardening Soil, está en la obtención de la cohesión, la dilatancia y el parámetro $m$, los cuales se deben determinar por medio de ensayos de laboratorio.

Los parámetros de peso unitario y ángulo de fricción obtenidos con técnicas avanzadas de exploración con el ensayo CPTu, son ligeramente mayores que los obtenidos mediante ensayos de laboratorio con técnicas convencionales de exploración.

El valor de Rigidez secante de ensayos tríaxiales se ajusta muy bien al encontrado por medio del registro del CPTu.
El perfil estratigráfico para el sector de la carrera 9a con calle 127 se caracteriza por tener parámetros de resistencia bajas.

El uso del ensayo CPTu debe de ir acompañado de algunos ensayos de laboratorio para validar la información obtenida.

\section{AGRADECIMIENTOS}

A la Universidad Pedagógica y Tecnológica por ser mi institución formadora.

\section{REFERENCIAS BIBLIOGRÁFICAS}

[1] Rodríguez Ordoñez J.A, Hacia la integralidad de la enseñanza y la práctica profesional en geotecnia, Revista Ingeniería y Universidad Pontificia Universidad Javeriana, 8(2) 159-171, ISSN 01232126, Julio-Diciembre 2004.

[2] Fondo de prevención y atención de emergencia, Estudio de zonificación de la respuesta sísmica de Bogotá para el diseño sismo resistente de edificaciones, Informe final, Vol 1., Santafé de Bogotá, Octubre 2010.

[3] Rodríguez Granados, E. E., Métodos modernos de exploración y caracterización del subsuelo. Memorias, curso de exploración y caracterización del subsuelo con técnicas avanzadas. Santafé de Bogotá, 1-92, Colombia, Mayo 2015.

[4] Brinkgreve, R.B.J, Reference Manual Plaxis 2D V8, Delft University of technology and Plaxis b.v, The Netherlands, 2004, pp. $1-152$.

[5] Instituto de desarrollo urbano IDU, Información Geotécnica PLBM Tramo IV, Disponible en: https://webidu.idu.gov.co/jspui/handle/123456789/2 432. [Citado el 7 de Julio de 2016].

[6] Rodríguez Granados, E. E., Ejemplos de aplicación del piezocono CPTu en proyectos en Colombia. Memorias, curso de exploración y caracterización del subsuelo con técnicas avanzadas. Santafé de Bogotá, 1-90, Colombia, Mayo 2015.

[7] Nieto Leal, A., Camacho Tauta, J.F. y Ruiz Blanco, E.F., Determinación de parámetros para los 
modelos elastoplásticos Mohr Coulomb y Hardening Soil en suelos arcillosos, Revista de Ingeniería Universidad de Medellín, 8(15) 75-91, ISSN 1692-3324, Julio-Diciembre de 2009.

[8] Robertson, P. K. \& Cabal Robertson K.L., Guide to cone penetration testing for Geotechnical Engineering, Gregg Drilling \& Testing. Inc., California, 2014.

[9] Marchetti, D. DMT y SDMT: Ejemplos de aplicación en proyectos. (Mayo 8: 2015: Santafé de Bogotá), Memorias, curso de exploración y caracterización del subsuelo con técnicas avanzadas. Santafé de Bogotá, 1-57, Colombia, Mayo 2015.

[10] Viggiani G. \& Atkinson J.H., Stiffnes of finegrained soil at very small strains, Géotechnique., 45(2) 249-265, ISSN 0016-8505, Junio 1995.

[11] Moya Barrios, J.E., Salazar Ferro, R., Gómez Agudelo, G.A. y Salazar Ferro F., Excavaciones En Condiciones Complejas, Escuela Colombiana De Ingeniería, Bogotá, 2014. 